\title{
Fluorescence Dynamic Anisotropy of Spinach Calmodulin Labeled by a Fluorescein Chromophore at Cys-26
}

\author{
Osamu Kogi, Atsuna Fukushima, Shoji Ishizaka, and Noboru Kitamura ${ }^{\dagger}$ \\ Division of Chemistry, Graduate School of Science, Hokkaido University, Sapporo 060-0810, Japan
}

(Received February 18, 2002; Accepted April 8, 2002)

\section{Introduction}

Calmodulin (CaM, 16.7 kDa and 148 amino acid residues) is an acidic protein expressed in all eukaryotic cells. It regulates cellular motility, ion transport, metabolism, and muscle contraction. ${ }^{1}$ Besides its biological roles, $\mathrm{CaM}$ is a very interesting protein since it possesses four $\alpha$-helix-loop- $\alpha$-helix subdomains (EF-hands I-IV from the protein N- to C-terimini) that bind four $\mathrm{Ca}^{2+}$ ions in total. The EF-hands I and II constitute a globular domain, which links with those of III and IV via a flexible $\alpha$-helix tether, leading to a dumbbell like shape of CaM. It is well known, furthermore, that CaM undergoes conformational changes upon binding of $\mathrm{Ca}^{2+}$. An observation of conformational changes of $\mathrm{CaM}$ by $\mathrm{Ca}^{2+}$ has been thus a very interesting target in spectroscopies. So far, fluorescentlylabeled CaM isolated from bovine brain, ${ }^{2}$ bovine testis, ${ }^{3,4}$ and wheat germ $^{5}$ has been studied on the basis of time-resolved fluorescence spectroscopy. Nonetheless, the results obtained by these studies are still controversial, since the number of the labeled-site in $\mathrm{CaM}$ is not necessarily one and this makes data analysis very difficult.

Recently, Bright and his co-workers reported spectroscopic studies of fluorescently-labeled spinach leaf $\mathrm{CaM}$ and demonstrated conformational changes of $\mathrm{CaM}$ by $\mathrm{Ca}^{2+} .6,7$ The advantage of using spinach leaf $\mathrm{CaM}$ is that the protein has only one cystein residue (Cys-26) and Cys-26 locates near the EFhand $\mathrm{I},{ }^{8}$ implying that one of the four $\mathrm{Ca}^{2+}$-binding sites can be selectively labeled by an appropriate probe. We think that a time-resolved single photon counting method could provide detailed information about conformational changes of CaM. Therefore, we explored picosecond fluorescence dynamics of fluorescein-labeled spinach leaf $\mathrm{CaM}(\mathrm{CaM}-\mathrm{F})$ in the presence and absence of $\mathrm{Ca}^{2+}$. Furthermore, it has been reported that ethanol can change conformations of the amino acid residues in CaM. ${ }^{7}$ Effects of ethanol on fluorescence dynamics of CaM-F are thus also an interesting target of the study.

\section{Experimental}

\section{Chemicals}

Fluorescein-5-maleimide (F5M, Molecular Probes), 3morpholinopropanesulfonic acid (MOPS, Dojin Chemicals), $O, O^{\prime}$-bis(2-aminoethyl)ethyleneglycol- $N, N, N^{\prime}, N^{\prime}$-tetraacetic

$\doteqdot$ To whom correspondence should be addressed. acid (EGTA) and Tris (both Pharmacia Biotech) were used as supplied. Other chemicals with the highest purity obtained from Wako Pure Chemicals were used without further purification. Distilled and deionized water (Toray, Toraypure LV-08) was used throughout the present study.

\section{Preparation of fluorescein-labeled CaM}

Calmodulin $(\mathrm{CaM})$ was isolated from spinach leaves on the basis of the reported procedures with some modifications. ${ }^{9}$ Siteselective labeling of spinach CaM with F5M at Cys-26 was conducted on the basis of the following reported protocols. ${ }^{6}$ Isolated spinach CaM was dissolved in a MOPS buffer $(10 \mathrm{mM}$ MOPS, $2 \mathrm{mM}$ EGTA, $0.5 \mathrm{M} \mathrm{NaCl}, \mathrm{pH}=7.0$ ). A five-fold molar excess amount of F5M in dimethyl sulfoxide was then added to the solution and the reaction was allowed to continue for $2 \mathrm{~h}$ in the dark at $\sim 23^{\circ} \mathrm{C}$. The reaction mixture was transferred to a dialysis cassette (MW cutoff $10 \mathrm{kDa}$, Pierce Chemical Company), and the solution was dialyzed exhaustively against the MOPS buffer until no fluorescence from free F5M was detected in the dialysate. After the dialysis, the solution containing fluorescein-labeled $\mathrm{CaM}(\mathrm{CaM}-\mathrm{F})$ was removed from the dialysis cassette, placed in a propylene carbonate microtube, sealed, and stored in the dark at $4^{\circ} \mathrm{C}$.

\section{Spectroscopic measurements}

Steady-state fluorescence spectroscopy was conducted by using a Hitachi F-4500 spectrofluorometer. Fluorescence decay measurements were conducted on the basis of a time-correlated single photon counting technique. ${ }^{10}$ By using a polarizer (Polaroid, HNP'B), the polarized direction of an excitation laser beam $(475 \mathrm{~nm})$ was set at a magic angle $\left(54.7^{\circ}\right)$ or vertical direction for fluorescence decay or dynamic anisotropy measurements, respectively. A polarizer (Polaroid, HNP'B) was set in front of a detector system for dynamic anisotropy measurements. Fluorescence from a sample was detected by a multichannel-plate photomultiplier tube (R3809U-50, Hamamatsu Photonics) equipped with a monochromator (CT$25 \mathrm{C}$, JASCO, monitor wavelength $=520 \mathrm{~nm}$ ) and analyzed by a single photon counting module (SPC-300, Edinburgh Instruments). All the experiments were conducted at room temperature $\left(\sim 23^{\circ} \mathrm{C}\right)$.

\section{Results and Discussion}

Steady-state fluorescence spectra of CaM-F

The fluorescence spectra of fluorescein-labeled spinach CaM 
$(2 \mu \mathrm{M})$ in the absence (apo-CaM-F) and presence of $3 \mathrm{mM}$ $\mathrm{CaCl}_{2}$ (holo-CaM-F) in a MOPS buffer exhibited analogous fluorescence characteristics with the maximum wavelength $\left(\lambda_{\max }\right)$ at around $515 \mathrm{~nm}$. Furthermore, the spectrum of fluorescein attached to CaM almost agreed with that of F5M itself $\left(\lambda_{\max }=514 \mathrm{~nm}\right)$. In the presence of ethanol (20 or 40 vol\%), on the other hand, the fluorescence intensity of CaM-F was weaker than that without ethanol. This was accompanied by a slight red shift of the spectrum $(517 \mathrm{~nm})$. Such a tendency was more pronounced at an ethanol content of $40 \%$ than in $20 \%$ ethanol. However, the fluorescence characteristics of CaM-F were almost identical, irrespective of the presence or absence of $\mathrm{Ca}^{2+}$ for a given ethanol content. These results indicate that the steady-state fluorescence properties of the fluorescein group attached to $\mathrm{CaM}$ are not sensitive enough to conformational change of the protein by $\mathrm{Ca}^{2+}$.

Doody et al. reported analogous effects of ethanol on the fluorescence characteristics of fluorescein-labeled spinach CaM. $^{7}$ In solution, fluorescein can exist as a dianion, monoanion, neutral, or cation form depending on solution $\mathrm{pH}$, ionic strength, or dielectric constant. Since fluorescein takes a neutral form in ethanol, ${ }^{11}$ they concluded that the fluorescence characteristics of CaM observed in the presence of ethanol were due to formation of the neutral form of the chromophore. Under the present experimental conditions in a MOPS buffer $(\mathrm{pH}=7)$, however, the primary form of the fluorescein chromophore in CaM-F is the dianion, as confirmed by the fluorescence ( 515 $\mathrm{nm})$ and absorption maximum wavelengths $(490 \mathrm{~nm})$. In the presence of ethanol (20 vol\%), the absorbance of holo-CaM-F at $490 \mathrm{~nm}$ decreased and this was accompanied by an increase in the absorbance of the monoanion $(460 \mathrm{~nm})$. Knowing the fluorescence quantum yields of the monoanion and dianion to be 0.37 and 0.93 , respectively, we conclude that the effects of ethanol on the fluorescence characteristics are due to the contribution of the monoanion.

\section{Fluorescence dynamic anisotropy of CaM-F}

We studied fluorescence dynamics of apo- and holo-CaM-F in a MOPS buffer with and without ethanol (20 or 40 vol\%). Apoand holo-CaM-F exhibited single exponential decays with the time constant of $\tau=4.2 \mathrm{~ns}$, while the fluorescence of F5M decayed double exponentially with $\tau_{1}=1.5 \mathrm{~ns}$ and $\tau_{2}=2.7 \mathrm{~ns}$. For CaM-F, it is worth noting that the fluorescence decay can be best analyzed by the single time constant of $\tau=4.1-4.2 \mathrm{~ns}$ irrespective of the ethanol content in a MOPS buffer.

The observation of the two time constants for F5M is very reasonable, since the molecule under the conditions exists in the two forms: dianion and monoanion. As judged from the amplitude of each decay component $\left(A_{1}\right.$ or $\left.A_{2}\right)$, the short $\left(A_{1}=\right.$ 0.23, $\left.\tau_{1}=1.5 \mathrm{~ns}\right)$ and long lifetime components $\left(A_{2}=0.77, \tau_{2}=\right.$ $2.7 \mathrm{~ns})$ are assigned as the monoanion and dianion of F5M, respectively. On the other hand, apo- and holo-CaM-F showed single exponential decays. It should be noted that Watkins and Bright reported that the fluorescence decay of fluorescein attached to CaM in a MOPS buffer in the presence or absence of $\mathrm{Ca}^{2+}$ could be analyzed by double exponential functions: $\tau_{1}=$ $2.0-2.6 \mathrm{~ns}\left(A_{1}=0.06-0.50\right)$ and $\tau_{2}=4.2 \mathrm{~ns}\left(A_{2}=0.94-0.50\right){ }^{6}$ In the presence of ethanol, they also reported that the fluorescence of CaM-F exhibited a double exponential decay. ${ }^{7}$ Although the observation of the time constant with $4.2 \mathrm{~ns}$ as the major component (judged from the $A_{2}$ value) agrees very well with the present results, their observation of the $\tau_{1}$ component contradicts with ours. Although we cannot compare the results by the Bright group and ours directly, the observation of the $\tau=$ 4.2 ns component in CaM-F indicates that the photophysical

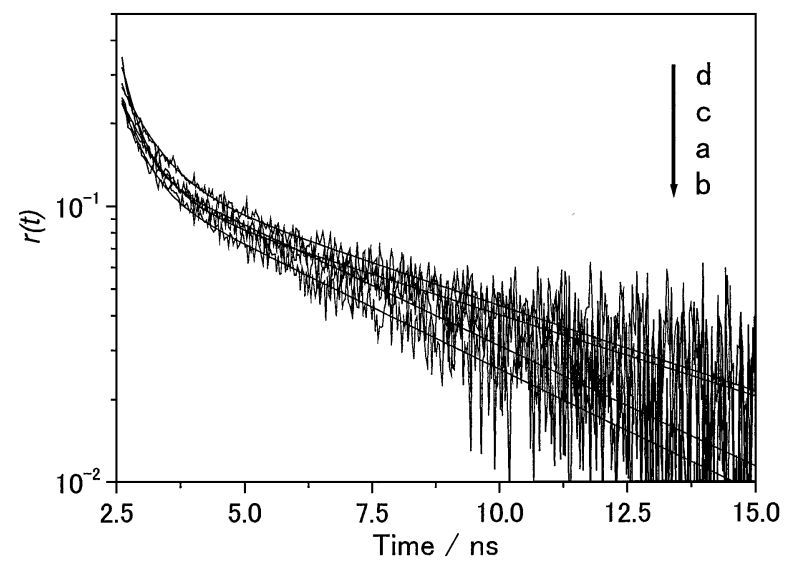

Fig. 1 Fluorescence anisotropy decay profiles of apo-CaM-F (a and b) and holo-CaM-F (c and d) in a MOPS buffer. The ethanol content $(\mathrm{vol} \%)$ in the solution was 0 (a and c) or $20 \%$ (b and d). The solid curves show the best fits by Eq. (1).

properties of the fluorescein group attached to Cys-26 in CaM are certainly affected by the presence of the amino acid residues composing the EF-hand I in CaM. Since the decay time is as long as $4.1-4.2 \mathrm{~ns}$, we suppose that the decay component can be ascribed to the excited state of the dianion of the fluorescein group in CaM-F. Microstructures around the fluorescein group in apo- and holo-CaM-F influence both the excited-state lifetime and the dianion-monoanion equilibrium of the chromophore. It is known that several basic amino acids exist in the EF-hands, ${ }^{12}$ so that these amino acids might stabilize the excited-state of the dianion, leading to relatively long excitedstate lifetimes in CaM-F.

Further detailed information about the structures of CaM-F and the microenvironments around the fluorescein group was obtained through fluorescence dynamic anisotropy. A fluorescence anisotropy decay of a probe molecule attached to a macromolecule provides direct information about motional dynamics of both the macromolecule (i.e., CaM-F) and the probe (i.e., fluorescein). The anisotropy decay, $r(t)$, is defined as in Eq. (1),

$$
r(t)=\left[I / /(t)-I_{\perp}(t)\right] /\left[I_{/ /}(t)+2 I_{\perp}(t)\right]=\sum r_{0}\left[A_{\mathrm{i}} \exp \left(-t / \tau_{\mathrm{i}}^{\mathrm{rot}}\right)\right]
$$

where $I / /(t)$ and $I_{\perp}(t)$ are the parallel and perpendicular components of the fluorescence decay, respectively. $r_{0}$ is initial anisotropy (i.e., $r(t)$ at $t=0)$ ). $\tau_{\mathrm{i}}^{\text {rot }}$ represents the $i$-th component of the rotational relaxation times and $A_{\mathrm{i}}$ is the amplitude relevant to $\tau_{\mathrm{i}}^{\text {rot. }}$. The anisotropy decays of apo- and holo-CaM-F in a MOPS buffer in the absence and presence of ethanol (20\%) are shown in Fig. 1. The observed parameters are summarized in Table 1. We found short $\left(\tau_{1}^{\text {rot }}=0.3-0.6 \mathrm{~ns}\right)$ and long rotational relaxation times $\left(\tau_{2}^{\text {rot }}=5-7 \mathrm{~ns}\right)$. It is clear from the $\tau_{1}^{\text {rot }}$ values that $\tau_{1}^{\text {rot }}$ and $\tau_{2}^{\text {rot }}$ are ascribed to those of the rotational motions of the fluorescein group in CaM-F and the whole of the CaM-F protein, respectively. It is worth noting that the $\tau_{2}^{\text {rot }}$ component is longer in the presence of ethanol (20\%) as compared with that without ethanol. Such results involve invaluable information about conformational changes of $\mathrm{CaM}$. Although the steady-state fluorescence characteristics are insensitive to the conformational changes of the protein, its dynamics are certainly sensitive enough to subtle structural changes in CaM. 
Table 1 Rotational reorientation parameters and calculated diameters of apo- and holo-CaM-F in MOPS buffer solution

\begin{tabular}{|c|c|c|c|c|c|c|c|c|}
\hline & Ethanol, \% & $A_{1}$ & $\tau_{1}^{\mathrm{rot} / \mathrm{ns}}$ & $A_{2}$ & $\tau_{2}^{\text {rot } / \mathrm{ns}}$ & $r_{0}$ & $V / \AA^{3 a}$ & $d / \AA^{\mathrm{a}}$ \\
\hline \multirow[t]{2}{*}{$\mathrm{CaM}-\mathrm{F},-\mathrm{Ca}^{2+b}$} & 0 & 0.21 & 0.27 & 0.14 & 5.0 & 0.35 & 19900 & 33 \\
\hline & 20 & 0.19 & 0.58 & 0.11 & 7.4 & 0.30 & 29700 & 38 \\
\hline \multirow[t]{2}{*}{$\mathrm{CaM}-\mathrm{F},+\mathrm{Ca}^{2+\mathrm{b}}$} & 0 & 0.19 & 0.43 & 0.12 & 4.9 & 0.31 & 19500 & 33 \\
\hline & 20 & 0.20 & 0.63 & 0.13 & 7.0 & 0.33 & 28200 & 38 \\
\hline
\end{tabular}

a. $V$ and $d$ denote the volume and diameter of the protein, respectively. b. $-\mathrm{Ca}^{2+}$ and $+\mathrm{Ca}^{2+}$ represent the absence and presence of $\mathrm{Ca}{ }^{2+}(3 \mathrm{mM})$ in the system, respectively.

\section{Conformation changes of CaM by ethanol}

$\tau_{2}{ }^{\text {rot }}$ is responsible for rotational reorientation of the protein, so that the value should provide information about the overall size and structures of CaM-F. One possible approach based on $\tau_{2}^{\text {rot }}$ is to estimate the volume of CaM-F $(V)$ by using a Debye-Stokes-Einstein model: $V=\tau_{2}{ }^{\text {rot }} R T / \eta$, where $R$ and $T$ are the usual meanings, and $\eta$ is the viscosity of the medium. We assume CaM-F to be a spherical shape. Knowing the $\tau_{2}^{\text {rot }}, R, T$ $(298 \mathrm{~K})$, and $\eta$ values $(1.02 \mathrm{cP})$, we calculated $V$ and the diameter of $\mathrm{CaM}(d)$ as the results were included in Table 1. The diameter of CaM in a MOPS buffer is $33 \AA$, while that in the presence of $20 \mathrm{vol} \%$ ethanol becomes $38 \AA$, which corresponds to an overall volume change of $\mathrm{CaM}$ of $\sim 45 \%$. It has been reported that a decrease in the medium polarity around $\mathrm{CaM}$ brings about conformational changes of the hydrophobic amino acid residues constituting the $\mathrm{Ca}^{2+}$ binding sites (EFhands I-IV) and, this allows exposure of the amino acids to the outer surface of CaM. ${ }^{12}$ Therefore, the present results on the volume change of $\mathrm{CaM}$ by an addition of ethanol could also be explained by the conformational change around the $\mathrm{Ca}^{2+}$ binding sites (EF-hands I-IV). By using the advantage of spinach $\mathrm{CaM}$ having only one cystein residue, an appropriate choice of a fluorescent probe will reveal further details about conformational changes of $\mathrm{CaM}$ by $\mathrm{Ca}^{2+}$.

\section{Acknowledgements}

The authors acknowledge Prof. Haeng-Boo Kim at The University of Tokyo for fruitful discussions. N. K. is also indebted to a Grant-in-Aid from the Ministry of Education,
Science, Sports, and Culture for the partial support of the research (No. 11440215) and O. K. thanks JSPS for a fellowship.

\section{References and Notes}

1. L. Van Eldik and D. M. Watterson (ed.), "Calmodulin and Signal Transduction", 1998, Academic Press, New York.

2. E. W. Small and S. R. Anderson, Biochemistry, 1988, 27, 419.

3. P. Bayley, S. Martin, and G. Jones, FEBS Lett., 1988, 238, 61.

4. I. Grycznski, R. E. Steiner, and J. R. Lakowicz, Biophys. Chem., 1991, 39, 69.

5. Y. Yao, C. Schöneich, and T. C. Squier, Biochemistry, 1994, 33, 7797.

6. A. N. Watkins and F. V. Bright, Appl. Spectrosc., 1998, 52, 1447.

7. M. A. Doody, G. A. Baker, S. Pandey, and F. V. Bright, Anal. Chem., 2000, 72, 227.

8. T. J. Lukas, D. B. Iverson, M. Schleicher, and D. M. Watterson, Plant Physiol., 1984, 75, 788.

9. D. M. Watterson, D. B. Iverson, and L. J. Van Eldik, Biochemistry, 1980, 19, 5762.

10. H.-B. Kim, S. Habuchi, and N. Kitamura, Anal. Chem., 1999, 71, 842 .

11. Z. Zhao, T. Shen, and H. Xu, Spectrochim. Acta, Part A, 1989, 45, 1113

12. M. Ikura, Tr. Biochem. Sci., 1996, 21, 14. 